\title{
Synthetic criticality in cellular brains
}

\author{
Ricard Solé ${ }^{2,3,6 *}$, Nuria Conde-Pueyo ${ }^{2,3}$, Antoni Guillamon ${ }^{1,4,5}$, \\ Victor Maull ${ }^{2,3}$, Jordi Pla ${ }^{2,3}$, Josep Sardanyés ${ }^{4}$, and Blai Vidiella ${ }^{2,3}$. \\ ${ }^{1}$ Departament de Matemàtiques, EPSEB, Universitat Politècnica de Catalunya, 08028 Barcelona, Spain \\ ${ }^{2}$ ICREA-Complex Systems Lab, Universitat Pompeu Fabra, 08003 Barcelona, Spain \\ ${ }^{3}$ Institut de Biologia Evolutiva (CSIC-UPF), 08003 Barcelona, Spain \\ ${ }^{4}$ Centre de Recerca Matemàtica. Edifici C, Campus de Bellaterra, 08193 Cerdanyola del Vallès, Barcelona, Spain \\ ${ }^{5}$ Institut de Matemàtiques de la UPC-BarcelonaTech (IMTech), \\ Universitat Politècnica de Catalunya, 08028 Barcelona, Spain \\ ${ }^{6}$ Santa Fe Institute, 1399 Hyde Park Road, Santa Fe NM 87501, USA
}

\begin{abstract}
Cognitive networks have evolved to cope with uncertain environments in order to make reliable decisions. Such decision making circuits need to respond to the external world in efficient and flexible ways, and one potentially general mechanism of achieving this is grounded in critical states. Mounting evidence has shown that brains operate close to such critical boundaries consistent with self-organized criticality (SOC). Is this also taking place in small-scale living systems, such as cells? Here we explore a recent model of engineered gene networks that have been shown to exploit the feedback between order and control parameters (as defined by expression levels of two coupled genes) to achieve a SOC state. We suggest that such SOC motif could be exploited to generate adaptive behavioral patterns and might help design fast responses in synthetic cellular and multicellular organisms.
\end{abstract}

Keywords: self-organised criticality, cell dynamics, brains, evolution, synthetic biology

\section{INTRODUCTION}

The relevance of criticality in complex systems is well exemplified by the dynamics exhibited by brains and brain cell cultures that is consistent with the presence of a self-organized critical state [1-4]. In this context, it was early conjectured that systems performing computations could highly benefit from operating close to phase transition points [5]. On the other hand, a dynamical view of such an advantage emerges from considering the need for fast responses to changing conditions in a fluctuating environment [6]. Flocks of birds and fish schools, for example, would also be organized as a class of active matter where local interactions operate in such a way that cohesive group order coexists with rapid collective responses stemming from external inputs such as the presence of predators [7-9]. Finally, criticality might also be a useful dynamical state to perform efficient collective tasks in ant and termite colonies to execute colony-level computations [10, 11].

While brain criticality is tied to the dynamics of large, interconnected networks of neurons, cognition itself is not limited to standard neural masses. At the other extreme of the complexity spectrum, the so called aneural systems, including in particular single-cell organisms, share a number of commonalities with complex neural agents. As it has been pointed out by several authors they can exhibit complex behavior thanks to arrays of receptors that gather information from the outside world and a no less complex network of downstream molecular cascades

\footnotetext{
*Corresponding author. ricard.sole@upf.edu
}

that provide the processing requirements to make decisions. By contrast with "solid" brains $[12,13]$ where the basic units (neurons) are located in given spatial locations and connected by a web of synaptic contacts, the inside of a cell (figure 1a) is a fluid, noisy and heterogeneous environment. Despite this apparent disorder, cells act as well-organized agents capable of properly and accurately respond to environmental uncertainty. This occurs thanks to the presence of information processing networks (figure 1b) that are, in many ways, not much different to well-known neural network models [14-16].

To some extent, we can talk about a "liquid" cellular brain where the process of information takes place in a spatially distributed manner under molecular interactions involving, as with neural networks, threshold-like functions. Early work based on microscopic observations of single cell organisms by Alfred Binet [17] and Herbert S. Jennings [18] supported the concept that some kind of cellular intelligence could be defined, particularly obvious in those tasks involving movement towards food or away from harmful toxins. Such behavioral patterns necessarily involve internal states, error perception (and correction) or learning. Beyond the metaphor, the neural network analogy has been explicitly suggested within the context of microbial behavior [19]. The differences between unicellular and multicellular organisms are considerable, starting from the fact that the former necessarily communicate by means of diffusion processes, whereas diffusion cannot be used as a reliable way of coordination in the later. The invention of neurons has much to do with the need for efficiently and directionally send messages across a cohesive, physically connected cell assembly. And yet, unicellular agents can also rapidly respond to external signals. 

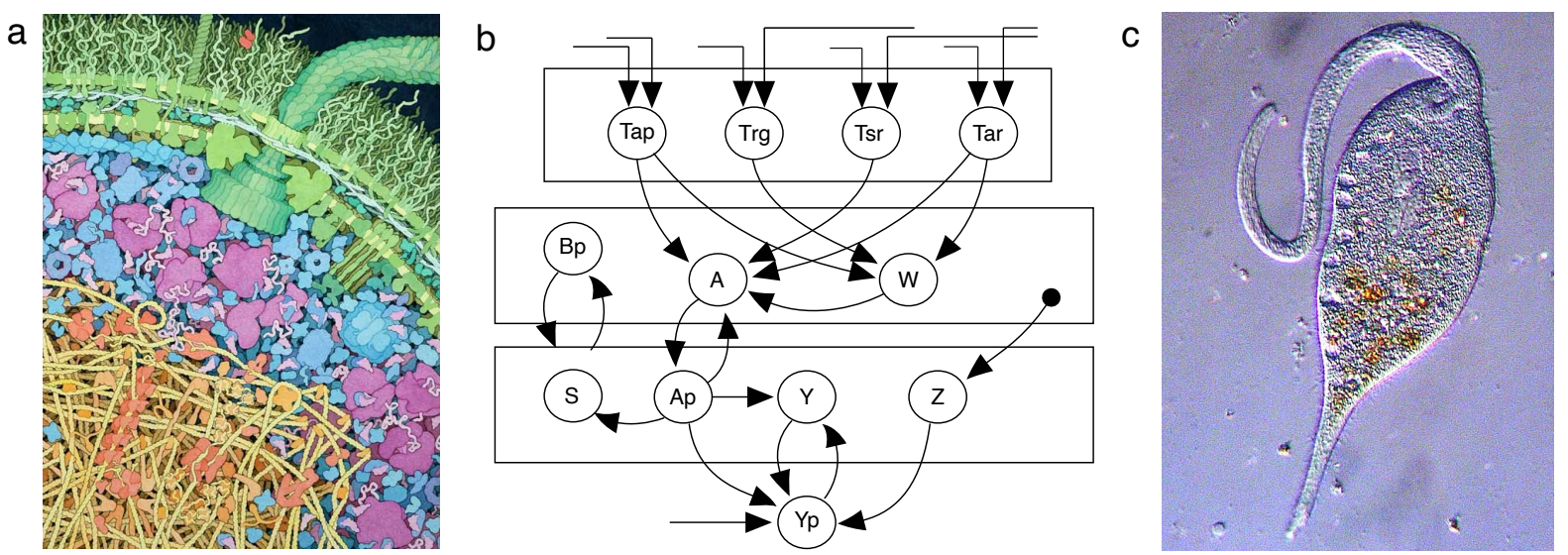

FIG. 1: The liquid "neural network" within cells. In (a) some details of the crowded cellular environment of the bacterium $E$. Coli are captured, including diverse macromolecules and a crucial "effector": the flagellum, that allows movement and search (image by David Goodsell, https://pdb101.rcsb.org/sci-art/goodsell-gallery/escherichia-coli). In (b) a stylized diagram of the downstream signaling cascades of a cell closely resembles a neural feedforward network (inspired in Bray, 1995). In (c) unicellular organisms such as Lacrymaria olor (a ciliate, image by Phil Gates, https://beyondthehumaneye.blogspot.com/2009/06/tearsof-swan.html) can display complex hunting strategies. The S-shaped structure is a flexible protrusion used to search and capture prey.

Since critical states seem to be present in cortical systems and play a functional role, it is worth considering the possibility that SOC might also be present in these fluid, cellular brains. In this paper we further explore this possibility, that has been recently raised in a study showing that SOC genetic circuits can be designed and implemented in living cells [20]. The origins of this SOC state are here analysed under a deterministic framework, showing that the model genetic circuit contains the right ingredients for a self-organized state, namely a feedback between order and control parameters, responsible for the generation of fat-tailed distributions of gene expression. Potential applications of this SOC circuit are suggested within the context of behavioural patterns and the evolutionary and computational implications are outlined.

\section{CELL CONGESTION: FREE AND JAMMED PHASES}

Our starting point makes a connection with one of the classic topics of SOC within statistical physics of complexity: traffic congestion. One of the classic examples of critical phenomena in complex systems is the NagelSchreckenberger model of freeway traffic [21-23]. This model revealed the presence of a phase transition between laminar flow and start-stop-waves separated by point of maximum flow separating the two phases. The model, despite its simplicity, was also shown to match real traffic behaviour. At criticality, wide fluctuations emerged. As discussed in this section, a similar scenario can be found (or engineered) within living cells.

How can bursting, self-organized critical states be generated inside single cells? The key requirement is to have a dynamical mechanism that exploits the tendency of a given genetic circuit to slowly increase gene expression levels (as grains of sand are added to a sandpile) until some negative feedback resets the state of the system. In order to create such a circuit, we start from a simpler model based on standard queueing transitions. In a previous study [24] it was shown how such a system can be defined, with the basic architecture as displayed in figure 2a. It involves the constitutive (i. e. constant, non-regulated) expression of a gene coding for a protein, labeled $\sigma$. The degradation of $\sigma$ takes place both spontaneously and as a result of the cellular proteolytic machinery [25], driven by means of the so called ClpXP protease (figure $2 \mathrm{~b}$ ). A simple model describing the dynamics of protein $\sigma$ follows a deterministic (mean-field) system, given by the differential equation:

$$
\frac{d \sigma}{d t}=\eta-\delta \sigma-\frac{\rho \sigma}{K+\sigma}=: f_{\mu}(\sigma)
$$

where $\mu$ indicates the set of parameters $(\eta, \delta, \rho, K)$. These parameters indicate: the efficiency $\eta$ of the (constitutive) expression of $\sigma$, its spontaneous decay rate $\delta$, the active degradation rate $\rho$ associated to the proteolytic machinery, and the Michaelis-Menten constant $K$. This model has a single attractor, namely

$$
\sigma_{+}=\frac{1}{2 \delta}\left(\eta-\rho-K \delta+\sqrt{(\eta-\rho)^{2}+K \delta(2 \eta+2 \rho+K \delta)}\right),
$$

which will always be stable since the sign of

$$
\lambda\left(\sigma_{+}\right)=\frac{d}{d \sigma} f_{\mu}\left(\sigma_{+}\right)=-\delta-\frac{\rho K}{\left(K+\sigma_{+}\right)^{2}}
$$

is always negative.

As we can see, this is not a standard phase transition (bifurcation) involving two fixed points whose stability 
a
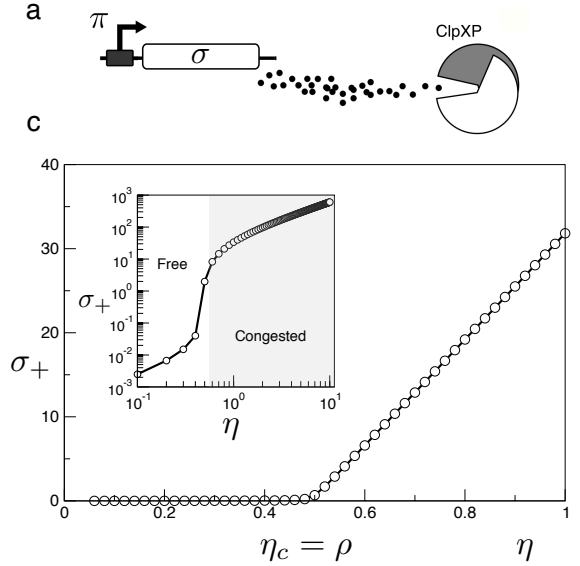

$\mathrm{b}$
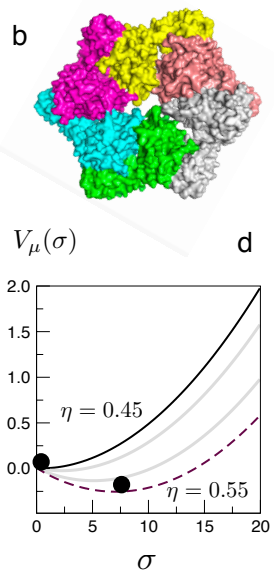

FIG. 2: Free and congested phases in a simple model of queueing dynamics in a genetic circuit. In (a) a single gene is constantly active and produces a protein $\sigma$ at a rate $\eta$ that is also actively degraded by a protease (ClpXP). If production is low, $\sigma$ levels are kept low, but there is a threshold level $\eta_{c}$ where the degradation machinery becomes overloaded and proteins accumulate, as summarized in (b). (c) The curve of equilibria of model (1) in terms of the rate $\eta$ displays an abrupt transition in the logarithmic scale near $\eta=\rho$. (d) Associated potential $V_{\mu}(\delta)$ for different $\eta$ values near the transition point.

is exchanged at criticality. Specifically, there is no phase involving an absorbing state, as it occurs with simple models of excitable dynamics [4]. This is summarized in figure $2 \mathrm{c}$, where we plot $\sigma_{+}$against $\eta$. In the linear scale plot the system apparently displays a continuous phase transition (a transcritical bifurcation) but it obviously does not (given the presence of a single fixed point) and this is displayed in the inset with the log-log plot. The reason for this is that the constant inflow of particles does not allow trivial (zero activity) states to occur.

These two regimes can be qualitatively inferred. For $K \rightarrow 0$ (a scenario relevant to the synthetic implementations) we have

$$
\left(\frac{d \sigma}{d t}\right)_{K \rightarrow 0} \approx \eta-\delta \sigma-\rho
$$

with a fixed point

$$
\sigma_{+}=\frac{\eta-\rho}{\delta}
$$

indicating that $\sigma_{+}$will be zero for $\eta<\rho$, and grow linearly as $\sigma_{+} \sim \eta / \delta$ with $\eta$. The non-critical character of the system is highlighted by looking at the associated potential $V_{\mu}(\sigma)$, defined from the condition $d \sigma / d t=-d V_{\mu}(\sigma) / d \sigma$ i. e. from

$$
V_{\mu}(\sigma)=-\int f_{\mu}(\sigma) d \sigma=(\rho-\eta) \sigma+\frac{\delta}{2} \sigma^{2}-\rho K \ln (K+\sigma)
$$

and displayed in figure $2 \mathrm{~d}$ for different values of $\eta$ close to the threshold value $\eta_{c}=\rho=0.5$. The shape of the potential does not allow to create the flattened valley close to $\eta_{c}$ and no fat-tailed distributions will be observed in the stochastic counterpart. In this context, although some other traits, such as latency times, do display scaling laws close to the $\eta_{c}$ boundary, no bursting avalanche dynamics will be observed. Can the presence of two distinct regimes be exploited to create a self-organized critical state? Work on statistical physics of self-organized critical traffic $[26,27]$ suggests a positive answer.

\section{SELF-ORGANIZED CRITICAL GENE NETWORKS}

The standard picture of self-organized criticality (SOC) incorporates the concept of a feedback loop between order and control parameters in such a way that a complex system can evolve towards a second-order phase transition [28-30]. In this self-organized regime, the propagation of interactions among system components can lead to power law distributions of events. Most of the time, the system operates under a low-activity basis but very large events can be triggered due to the intrinsic excitability that is characteristic of criticality. As a result, SOC states would pervade a number of adaptive properties, including fast responses and maximal information processing. Here a critical point is an "attractor", meaning that the system poises itself close to the transition boundary. In the canonical picture (such as the rice pile) as the system enters into the dynamical regime where avalanches are generated, the order parameter will exhibit critical fluctuations [31,32]. In this case, it would be the angle of repose $\theta_{c}$ (as discussed in Ref. [20]). Each time an avalanche occurs, the slope $\theta$ can decrease below the critical $\theta_{c}$ control parameter, which will then start to grow back towards $\theta_{c}$ as new sand grains are slowly added to the pile.

Are there genetic circuits within cells operating in a SOC state? Could such dynamical states have played a key role in evolving cell-level cognitive complexity? Transcriptional bursting has been shown to play a relevant role in eukaryotic systems across multiple scales [34]. Here bursting allows information transmission in a digital fashion, while regulatory strength is encoded in the frequency of the fluctuations, although not necessarily connected to criticality. Some indirect observations suggest that SOC dynamics might occur in enzymatic networks [33]. An alternative path to explore these questions is to engineer SOC circuits using synthetic biology, as the one explored here [20] that allows to obtain SOC from the previous single-gene congestion model.

\section{A. SOC network motif: deterministic properties}

The basic architecture of the SOC motif is displayed in figure 3a. The first gene expressing $\sigma_{1}$ is now degraded in competition with another protein $\sigma_{2}$ coded by another gene. Both genes are constitutively expressed: they are 

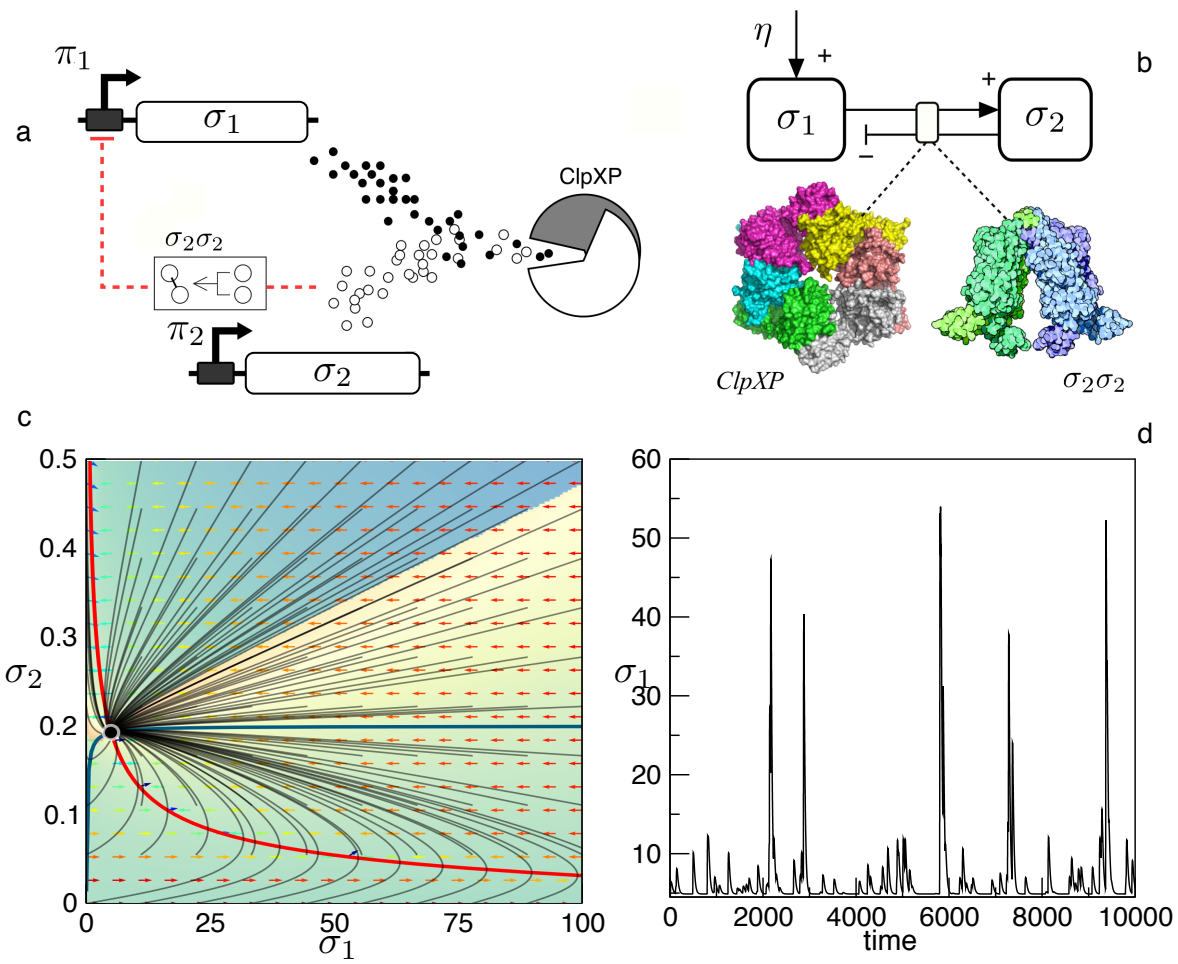

FIG. 3: The SOC genetic motif that generates fat-tailed distributions of protein expression levels. The basic architecture (a) includes two genes whose protein products will play the role of order $\left(\sigma_{1}\right)$ and control $\left(\sigma_{2}\right)$ paremeters, respectively. The basic feedbacks between the two are sketched in (b) where two molecular models of the proteolytic machinery (ClpXP) and the inhibitor dimer $\left(\sigma_{2} \sigma_{2}\right)$ are shown (images generated with Pymol software). In (c) the nullclines and the corresponding vector field of SOC motif is shown for the set $\Lambda_{0}$ using $\eta_{2}=0.01$. In (d) a time series of $\sigma_{1}$ is displayed, with $\sigma_{2}$ randomly perturbed in a small amount $\sigma_{2}=\sigma_{2}^{*}-\xi$ where $\xi \in(0,0.1)$ with uniform distribution. Bursting dynamics in $\sigma_{1}$ are observed.

constantly expressing their respective proteins at rates that are defined by the binding properties of their promoters $\left(\pi_{1}\right.$ and $\left.\pi_{2}\right)$. The efficiency of protein production can be properly tuned to guarantee that concentrations of both gene products are enough for the motif to be functional. Additionally, when the concentration is high enough, the protein monomers $\sigma_{2}$ form dimers, indicated as $\sigma_{2} \sigma_{2}$. Under this form, the protein complex represses the expression of $\sigma_{1}$. This implies a threshold condition: while concentrations are low, no dimers will be formed, but once congestion starts to emerge, $\sigma_{2}$ acts as a proper controller, reducing the expression of $\sigma_{1}$ to low levels. In this way we introduce the required negative loop from control to order parameter [35]. This is summarized in figure $3 \mathrm{~b}$ where the qualitative nature of the interactions is indicated. Here, the constitutive production of $\sigma_{1}$ (the order parameter gene) acts as the constant driving that pushes up the expression level of this protein. Its increase causes congestion and raises the levels of $\sigma_{2}$ (since it cannot be properly degraded). As a consequence, dimers get formed and the negative feedback emerges.

This theoretical design was experimentally implemented using E. coli strains and fluorescent proteins (Green and Red Fluorescent Proteins, GFP and RFP, respectively) were used as reporters to track the expres- sion levels of both $\sigma_{1}$ and $\sigma_{2}$. It was shown that, under a broad range of conditions, the feedback described here was able to generate a population-level pattern consistent with a SOC state. Using standard genetic engineering tools, the engineered parts were introduced in a single plasmid and the expression levels for each cell gathered by means of flow cytometry [20]. From these data sets, the levels of GFP displayed by a population of bacterial cells at a given time were shown to be power-law distributed. The design principle adopted in this motif is captured by a two-dimensional system of nonlinear ordinary differential equations describing the coupling between the order $\left(\sigma_{1}\right)$ and the control $\left(\sigma_{2}\right)$ parameters required to obtain criticality:

$$
\begin{array}{r}
\frac{d \sigma_{1}}{d t}=f\left(\sigma_{2}\right)-\delta_{1} \sigma_{1}-\sigma_{1} \Gamma\left(\sigma_{1}, \sigma_{2}\right), \\
\frac{d \sigma_{2}}{d t}=\eta_{2}-\delta_{2} \sigma_{2}-\sigma_{2} \Gamma\left(\sigma_{1}, \sigma_{2}\right),
\end{array}
$$

where the following Hill function response [35] is used:

$$
f\left(\sigma_{2}\right)=\frac{\eta_{1}}{\theta+\sigma_{2}^{2}},
$$

for the repression mediated by $\sigma_{2} \sigma_{2}$ dimers. When $\sigma_{2}$ is small (the ClpXP system is working far from congestion) 
we have a production rate $f\left(\sigma_{2} \rightarrow 0\right) \approx \eta_{1} / \theta$. The inhibition function has a threshold value $\theta$ representing the concentration $\sigma_{2}^{*}$ at which the rate drops to half its maximum value i. e. $f\left(\sigma_{2}^{*}\right)=\eta_{1} /(2 \theta)$. For larger values, it rapidly decays to zero. The saturation function, namely:

$$
\Gamma\left(\sigma_{1}, \sigma_{2}\right)=\frac{\delta_{c} C}{K+\sigma_{1}+\sigma_{2}},
$$

introduces the competition of both proteins for the proteolytic machinery. When no congestion occurs (due to low concentrations of both $\sigma_{1}$ and $\sigma_{2}$ ) we obtain a constant removal rate proportional to the concentration of ClpXP units, i. e. $\Gamma(0,0)=\delta_{c} C / K$.

The SOC motif model displays (as desired) a single attractor within the meaningful domain defined by the non-negative concentration space, namely

$$
\Omega_{1}=\left\{\left(\sigma_{1}, \sigma_{2}\right): \sigma_{1} \geq 0, \sigma_{2} \geq 0\right\} .
$$

Intuitively, we can see that the saturation term forbids the possibility that the system grows in unbounded ways, whereas the input terms do not allow "exclusion states" (with one of the species vanishing). The single fixed point $\left(\sigma_{1}^{*}, \sigma_{2}^{*}\right)$ is obtained from the intersection of the nullclines obtained from $d \sigma_{1} / d t=d \sigma_{2} / d t=0$ (see figure $3 \mathrm{c}$ ). Although we have several parameters, as defined by the parameter set

$$
\boldsymbol{\Lambda}:=\left(\eta_{1}, \eta_{2}, \theta, \delta_{1}, \delta_{2}, \delta_{c}, C, K, \mu\right) .
$$

In our previous work [20], we fixed most of the parameters accordingly to well established estimates. Here we use the same data set with the specific values: $\boldsymbol{\Lambda}_{0}=$ $\left(10^{-2}, \eta_{2}, 10^{-3}, 5 \cdot 10^{-2}, 5 \cdot 10^{-2}, 10^{-1}, 10^{-1}, 10^{-3}, 1\right)$. The behaviour was shown to be rather robust, with parameter $\eta_{2}$ requiring a minimal level in order to guarantee that the inhibitory regulation works. This can be analyzed by computing the eigenvalues of the Jacobian $J\left(\sigma^{*}\right)$ of our SOC motif system, given by

$$
J\left(\sigma^{*}\right)=\left(\frac{\partial \dot{\sigma_{i}}}{\partial \sigma_{j}}\right)_{\sigma^{*}}=\left(\begin{array}{cc}
-\delta_{1}-\Gamma+\sigma_{1} \Gamma & \frac{-2 \eta_{1} \sigma_{2}}{\left(\theta+\sigma_{2}^{2}\right)^{2}}+\sigma_{1} \Gamma \\
\sigma_{2} \Gamma & -\delta_{2}-\Gamma+\sigma_{2} \Gamma
\end{array}\right)_{\sigma^{*}} .
$$

with $\Gamma=\Gamma\left(\sigma_{1}, \sigma_{2}\right)$. These eigenvalues have always a negative real part, and they also display interesting properties as $\eta_{2}$ is increased [20]. In particular, it was shown that some statistical properties displayed by the stochastic counterpart (see below) were captured by the deterministic description. An example is the presence of a maximal variance in the distribution that occurs at a given $\eta_{2}^{c}$ rate and that is connected with the transition between stable node and stable focus.

The shape of the nullclines and the underlying vector field allow to predict that variations in the control parameter $\sigma_{2}$ can cause wide changes in the order parameter $\sigma_{1}$, as desired. Starting from the fixed point $\sigma^{*}$, perturbations in $\sigma_{1}$ push the system within a domain of the phase portrait where a rapid growth in $\sigma_{1}$ occurs before it returns back to equilibrium. An example of this is shown in figure $3 \mathrm{~d}$, where random, finite shifts in $\sigma_{2}$ are introduced at random times using the parameters from the set $\boldsymbol{\Lambda}_{0}$ and $\eta_{2}=0.01$. Fast, broad fluctuations are clearly appreciated. What is the statistical pattern displayed by these fluctuations?

\section{B. Genetic avalanches: stochastic dynamics}

The experimental implementation of the previous motif (with some specific additions required to properly test the model and its parameter dependencies) revealed that power laws in the expression pattern of $\sigma_{1}=[\mathrm{GFP}]$ are indeed observable. Here the state of each cell is gathered from a cell population sample (after a transient time) and the cells flow (one cell at a time) through a laser beam. Thanks to the specific fluorescent label (green and red, in our case) light is absorbed and then emitted in a band of wavelengths and the intensity of each selected wavelength measured for each cell.

Cumulative plots were used: if $P\left(\sigma_{1}\right)$ indicates the probability distribution, the cumulative distribution is defined as $P_{>}\left(\sigma_{1}\right)=\int_{0}^{\sigma_{1}} P(\sigma) d P(\sigma)$. For our system, we obtained $P_{>}\left(\sigma_{1}\right) \sim \sigma_{1}^{-2}$. Since the time scale for cell division is short (comparable with the bursting dynamics scale) no time series were available, and thus we implicitly assume ergodicity: the time snapshot provided by the Flow Cytometry data should match the time evolution of the system. An example of these distributions is shown in figure $4 \mathrm{a}$.

The experimental results matched the predicted statistics obtained from the stochastic version of the SOC motif, as displayed in figure $4 \mathrm{a}$. The model described above can be stochastically implemented using a Gillespie algorithm [36]. The result reveals that, once the concentration required for $\sigma_{2}$ (the feedback loop from the control parameter) reaches a sufficiently high threshold, gene expression avalanches occur resulting in a power law [20], as the one shown in figure $4 b$ that gives the same distribution. These fat-tailed distributions actually become dominant in a very large domain of the parameter space.

The spontaneous tendency towards criticality can be illustrated by considering a further simplification of the dynamical model. Consider the approximation $d \sigma_{2} / d t \approx$ 0 , i. e. that its dynamics if much faster than the one associated with the order parameter. In this case, we can re-write the SOC motif dynamics as follows:

$$
\frac{d \sigma_{1}}{d t}=\hat{\eta}_{1}-\delta_{1} \sigma_{1}-\sigma_{1} \frac{\delta C}{K+\sigma_{1}+\sigma_{2}}
$$

where we introduce a time-dependent function:

$$
\hat{\eta}_{1}=f\left(\sigma_{2}\right)=\frac{\eta_{1}}{\theta+\sigma_{2}^{2}} .
$$

This captures the changes in the fluctuations in the driving rate associated with the order parameter resulting 

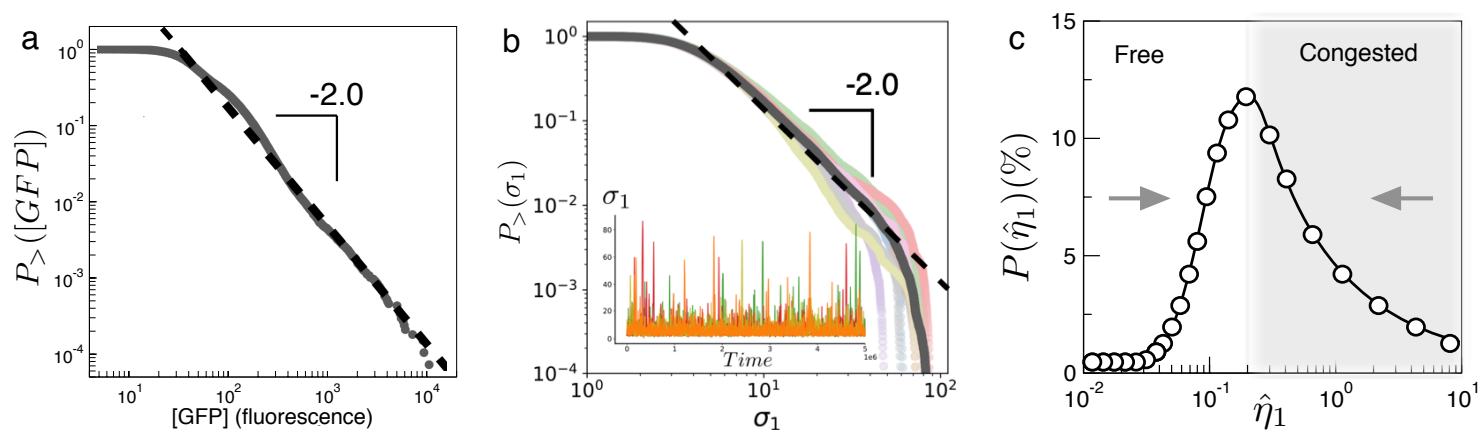

FIG. 4: Statistical patterns of fluctuations of the SOC motif. The experimental (a) and stochastically simulated (b) cumulative distributions consistently reveal a power-law behavior following the same exponent. The inset in (b) shows a time series of $\sigma_{1}$ fluctuations. In (c), the self-organized nature of the SOC motif can be visualized by means of the distribution of the effective $\eta_{1}$, here indicated as $\hat{\eta}_{1}$, as defined by equations (12-13). The distribution shows that this quantity peaks at a given value $\hat{\eta}_{1} \sim 0.02$, close to the mean field result found in the queueing gene model (section 2 ). The two opposed forces, namely the driving towards higher $\sigma_{1}$ expression and the $\sigma_{2}$-mediated repression, are displayed (gray arrows).

from the functional form associated to the control parameter. As defined, we have in fact transformed the original two-dimensional model into a self-organized congestion model (similar to the one discussed before) where $\eta_{1}$ is now a self-tuned parameter that keeps adjusting itself as $\sigma_{2}$ fluctuates. By following the values of $\hat{\eta}_{1}$ and computing their frequency, a new distribution is obtained, and shown in figure 4c. As expected for a SOC system, it shows a peak close to the boundary found for the single-gene queueing model, separating the free from the congested behavior except that, in this case, not such boundary exists.

The engineering of SOC dynamics within living cells provides a proof of concept for the design of reliable gene circuits that can generate this fat-tailed class of noise. This is a first step that promises to expand our potential for designing nonlinear phenomena with features similar to those found in neural systems. How can this SOC motif principle be applied to tune cell behavior?

\section{USING CRITICALITY TO CONTROL CELLULAR BEHAVIOR}

In order to adapt to environmental challenges, biological systems exhibit a diverse array of response mechanisms grounded in sensors and actuators as well as in information-processing units. Adaptive responses require dynamical features that combine low energetic costs along with fast changes to efficiently respond to environmental changes. Within cells, noise was early identified as playing multiple roles affecting cell fate, population heterogeneity, signal amplification or response to stress [37-39]. Noise is both an inevitable outcome of stochastic molecular interactions and an essential ingredient in decision making [40]. Several features of cell dynamics might result from criticality. One example is the complex dynamics of microtubules in eukaryotic cells [41-44]. In this case, the length and spatial distribution of these structures determine cell's shape and are crucial to cell motility, division and intracellular transport. Both measured changes in microtubule length and their explanation in terms of statistical physics models support the concept that they operate in a dynamical instability state. In this, coevolution has acted as the mechanism that tuned the rates of molecular assembly and disassembly.

As pointed out at the beginning of this paper, singlecelled organisms possess a broad repertoire of behavioral responses that allow them to navigate in changing environments. Bacteria such as E. coli detect and react to chemical gradients while paramecia or amoeba (Eukaryotic organisms) are capable of rapidly reacting to currents and obstacles. Using criticality as a way of rapidly responding to stimuli could enhance the efficiency of such responses. In what ways our SOC motif could be used to create or modify cognitive tasks?

One possible way of using our SOC motif as a way to tune microbial behaviour could involve a coupling between the two-gene network and the cellular machinery responsible for movement [45]. This machinery, as defined by a signaling network, is particularly well known in E. coli, where a group of signaling proteins are involved in the sensing of environmental cues, processing these as input signals and eventually acting on the flagella motor that is responsible for cell movement [46] (see figure 5). Specifically, the bacterium has a default direction of rotation, which is counterclockwise in E. coli. The flagellar motor always rotates counterclockwise unless the appropriate signal is sent. A group of signal transduction proteins $\{C h e A, C h e Y, C h e Z \ldots\}$ are involved. These proteins form a network motif $\phi$ (figure $5 \mathrm{a}-\mathrm{b}$ ) that includes the sensor, processing and effector steps required to connect environment and cell displacement. The concentrations of $C h e Y$ and $C h e Z$, in particular, are known to affect swimming behavior. High concentrations of an active form of ChezY (indicated as CheY-P in figure 5a) 


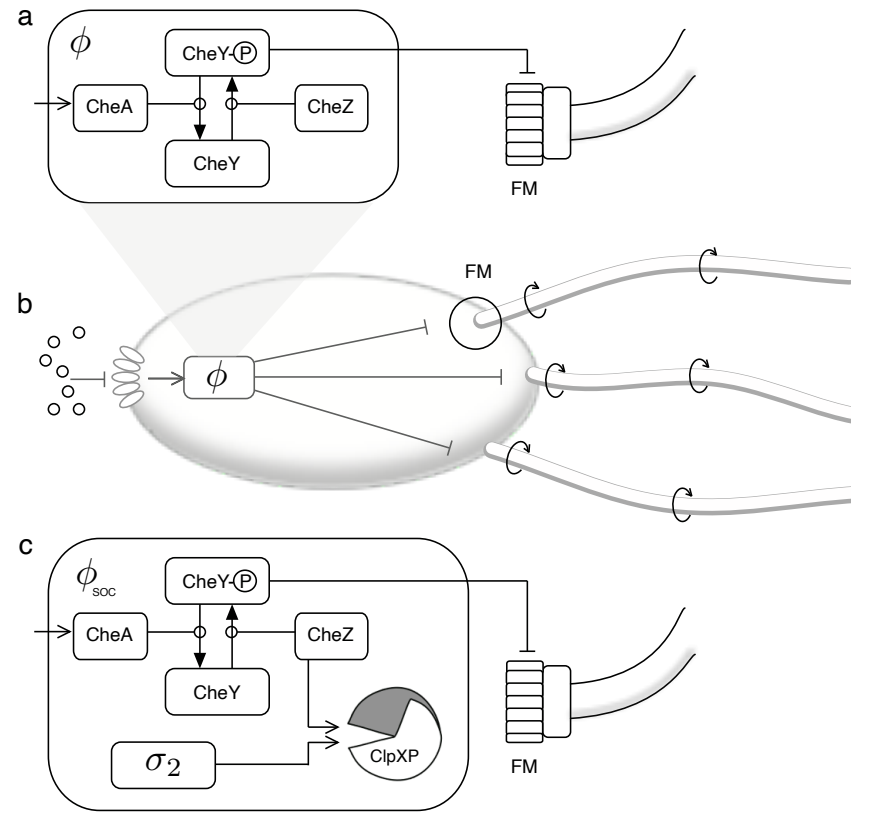

FIG. 5: Using synthetic SOC to modify cell-level search behaviour. A network $\phi$ (a) gathers signals from the environment (such as resources, b) that trigger a response affecting flagellar motor (FM). The $\phi$ network could be expanded into a new one $\left(\phi_{s o c}\right)$ by engineering the degradation of CheZ (c), a key component of this machinery.

enhance tumbling whereas CheZ inhibits ChezY. As a consequence, we conjecture that using CheZ as our $\sigma_{1}$ component could allow to tune bacterial chemotaxis.

The proposed extension of the SOC motif is summarized in figure $5 \mathrm{c}$ : using $\mathrm{CheZ}$ as the order parameter and the SOC motif design for its control, the new circuit $\phi_{\text {soc }}$ would produce bursts in CheZ that should modify the pattern of flagella rotation. By tuning $\eta_{2}$, we would expect to see different movement patterns. This is an interesting scenario to test ideas concerning the search patterns of organisms that take advantage of given statistical regularities of fat-tailed distributions of jumps. If such tuning works, it would be possible to engineer different strains and perform competition experiments to test the hypothesis that critical dynamics can be an optimal solution to explore heterogeneous environments. This is a specially active area within the physics of complex systems known as active matter [47]. In general terms, this is a novel class of non-equilibrium materials made of a large number of energy-dependent units that display collective behavior. The study of the motion of living swarms in particular has been largely inspired by the study of the motion of ants, termites and microorganisms [48]. Swarms are known to display critical dynamics too, and our synthetic design could help explore the potential phases exhibited by possible (but not actual) classes of behaviour. This will be particularly relevant if the SOC motif is extended towards cell-cell communication patterns.

Another application of the critical gene network concerns the engineering of bacteria used in some biomedical applications such as the detection and elimination of cancer cells $[49,50]$, a very active area within synthetic biology, which has taken advantage of nonlinear dynamical phenomena such as synchronization [51]. The key concept is that tumor-selective expression of a given gene can be placed under the control of some external signal that is associated to the presence of tumor populations. Examples of such signals characteristic of many tumors are the presence of hypoxia (i. e. reduced oxygen levels) or glucose gradients often found in solid tumors. Once the bacterium senses the right conditions (which could be threshold-level triggers) antitumour proteins can be expressed close to metabolically active regions of the tumor mass. Having a SOC motif coupled to such as sensoractuator system would ensure a highly sensitive targeting of cancer cells. If such external drivers (perhaps affecting our candidate $\sigma_{1}$ factor) are absent, low levels of delivery of therapeutic payloads would be expected, thus limiting potential cytotoxic effects on normal tissue. As soon as the engineered cell gets closer to the target tumor, the high excitability of the SOC motif would lead to bursting episodes of release, which can be tied to cell death. Under this scenario, as cells burst, they deliver the anticancer agents.

There is a potentially broad range of situations where our critical motif can play a useful role closer to the one found in neural networks. As we mentioned at the beginning, cellular circuits have been compared to neural networks, and the rich complexity of cellular webs should offer multiple possibilities to exploit our design principle.

\section{DISCUSSION}

The idea that complex patterns of cellular behavior can be an emergent property of gene-gene interactions was early proposed by Stuart Kauffman, who used a Boolean approximation $[52,53]$. This view was originated shortly after the classical work by Jacques Monod and François Jacob on the switching behaviour of gene regulation [54]. It is interesting to notice that, since those early years, both neural and genetic networks have received increasing attention both at the level of the details of their interacting constituents as well as in terms of theoretical models. In both cases, response functions are typically stepwise. One particularly key result from the study of Boolean gene network dynamics was the presence of a critical transition associated to the attractor dynamics of differentiate states. It was found that a critical connectivity separating an ordered and a disordered phase allowed a limited set of robust cell states to exist [55-57]. As a consequence of this prediction, perturbations of genes should trigger cascades of changes affecting $s$ genes following a critical branching process scaling $P(s) \sim s^{-3 / 2}$, as confirmed by knock-out data. Our SOC motif offers a 
new layer of complexity to this picture of cells as systems poised close to criticality.

Are SOC motifs likely to be present in cells? The basic components of the SOC design presented here are widespread in natural genetic regulatory networks. Many proteins are processed by the proteolytic machinery and most regulatory feedbacks involve dimerized complexes following the functional responses described above. The kind of critical circuit that we have discussed here should not only be possible: We conjecture that it might be present and future work should explore potential candidates and their potential relevance.

Our design principle could be translated into the multicellular domain. We have limited ourselves to single-cell dynamics, but engineering communication between cells is also easy to achieve and pervades the development of collective synthetic intelligence [58]. In this context, it has been shown that some simple (non-neural) organisms, such as Placozoans, exhibit criticality in their locomotion dynamics [59, 60]. Such kind of pattern could be also engineered in mammalian cells using our basic design principle. One candidate to this implementation are so called bio-robots $[61,62]$ which have been created by means of the assembly of different cell types from amphibians. Here one of the cell populations spontaneously exhibits excitable dynamics that allows the synthetic organisms to move in simple ways. Could a SOC motif including cell-cell communication allow them to display complex, adaptive search patterns closer to their natural counterparts? This possibility opens new avenues in the exploration of so called synthetic evolutionary transitions [63] and the understanding of the origins of early forms of cognition.

\section{ACKNOWLEDGMENTS}

The authors thank the members of the Complex Systems Lab, particularly Luis Seoane and Jordi Piñero for fruitful discussions. This work was supported by the Spanish Ministry of Economy and Competitiveness, grant PID2019-111680GBI00, an AGAUR FI 2018 grant, and the Santa Fe Institute (where the key idea was first conceptualized at the Cormac McCarthy's library). JS has been partially funded by the CERCA Programme of the "Generalitat de Catalunya", by "Agencia Estatal de Investigación" grant RTI2018-098322-BI00 and by the "Ramón y Cajal" contract RYC-2017-22243. AG has been funded by the AGAUR grant 2017-SGR-1049 and by the MINECO-FEDER-UE grants PGC-2018-098676B-100 and RTI2018-093860-B-C21. JP was funded by FPI 2020 .

\section{REFERENCES}

[1] Chialvo, D.R., 2004. Critical brain networks. Physica A 340, 756-765.

[2] Chialvo D.R. 2010. Emergent complex neural dynamics. Nat. Phys. 6(10), 744-750.

[3] Plenz D., Niebur E., Schuster H.G. Criticality in neural systems. Wiley-VCH, Weinheim, Germany (2014).

[4] Hesse J., Gross T. 2014. Self-organized criticality as a fundamental property of neural systems. Front. Neurosci. 8, 166.

[5] Langton, C. G. 1990. Computation at the edge of chaos: phase transitions and emergent computation. Physica D $42,12-37$.

[6] Mora T., Bialek W. 2011. Are biological systems poised at criticality? J.Stat. Mech. 144, 268-302.

[7] Bartumeus, F., 2007. Lévy processes in animal movement: an evolutionary hypothesis. Fractals,15, 151-162.

[8] Bialek, W. et al. (2014) Social interactions dominate speed control in poising natural flocks near criticality. Proc. Natl. Acad. Sci. U. S. A. 111, 7212-7217

[9] Couzin, I.D., 2018. Synchronization: the key to effective communication in animal collectives. Trends in cognitive sciences, 22, 844-846.

[10] Solé R., Miramontes O. 1995. Information at the edge of chaos in fluid neural networks. Physica D 80, 171-180.

[11] Miramontes O., 1995. Order-disorder transitions in the behaviour of ant societies. Complexity 1, 56-60.

[12] Solé, R., Moses, M. And Forrest, S., 2019. Liquid brains, solid brains. Phil. Trans. R Soc. B374, 20190040.

[13] Pinero, J. and Solé, R., 2019. Statistical physics of liquid brains. Phil. Trans. R Soc. B374, 20180376.

[14] Bray, D., 1990. Intracellular signalling as a parallel distributed process. J. Their. Biol. 143, 215-231.

[15] Bray, D., 1995. Protein molecules as computational elements in living cells. Nature 376, 307-312.

[16] Bray, D., 2009. Wetware: a computer in every living cell. Yale University Press.

[17] Binet, Alfred, 1888. The Psychic Life of Microorganisms. Open Court, Chicago.

[18] Jennings, H.S., 1905. Behavior of the Lower Organisms. Indiana University Press.

[19] Armitage, J.P., Holland, I.B., Jenal, U. and Kenny, B., 2005. "Neural Networks" in Bacteria: Making Connections. J. Bacterial. 187, 26-36.

[20] Vidiella, B., Guillamon, A., Sardanyés, J., Maull, V., Conde, N. and Solé, R., 2021. Engineering self-organized criticality in living cells. Nat Commun 12, 4415 (2021). https://doi.org/10.1038/s41467-021-24695-4

[21] Nagel, K. and Schreckenberger, M. J. 1992. A cellular automaton model for freeway traffic. Journal de Physique I. 2, 2221-2229.

[22] Nagel, K. and Rasmussen, S. 1994. Traffic at the edge of chaos. In: R. A. Brooks and P. Maes, editors, Artificial Life IV, pp. 222-235. MIT Press, Cambridge, MA.

[23] Helbing, D., 2001. Traffic and related self-driven manyparticle systems. Rev. Mod. Phys. 73, 1067.

[24] Cookson, N.A., Mather, W.H., Danino, T., Mondragon, et al. 2011. Queueing up for enzymatic processing: correlated signaling through coupled degradation. Mol. Sys. 
Biol. 7, 561

[25] Gottesman, S., 2003. Proteolysis in bacterial regulatory circuits. Annu. Rev. Dev. Biol. 19, 565-587.

[26] Solé R. and Valverde, S., 2001. Information transfer and phase transitions in a model of internet traffic. Physica A 289, 595-605.

[27] Guimerà, R., Díaz-Guilera, A., Vega-Redondo, F., Cabrales, A. and Arenas, A., 2002. Optimal network topologies for local search with congestion. Phys. Rev. Lett. 8, 248701.

[28] Sornette, D., Johansen, A. and Dornic, I., 1995. Mapping self-organized criticality onto criticality. Journal de Physique I, 5, 325-335.

[29] Jensen, H.J., 1998. Self-organized criticality: emergent complex behaviour in physical and biological systems. Cambridge U. Press.

[30] Muñoz M.A. 2018 Colloquium: criticality and dynamical scaling in living systems. Rev. Mod. Phys. 90, 031001.

[31] Bak, P., C. Tang, and K. Wiesenfeld. 1988. Self-organized criticality. Phys. Rev. A 38, 364?374.

[32] Christensen, K. And Moloney, N. Complexity and Criticality. Imperial College Press, London. 2005.

[33] Steiner, P.J., Williams, R.J., Hasty, J. and Tsimring, L.S., 2016. Criticality and adaptivity in enzymatic networks. Biophysical journal, 111, 1078-1087.

[34] Wang, Y., Ni, T., Wang, W. and Liu, F., 2019. Gene transcription in bursting: a unified mode for realizing accuracy and stochasticity. Biological Reviews 94, 248258.

[35] Alon, U., 2019. An introduction to systems biology: design principles of biological circuits. CRC press.

[36] Gillespie D. T. 1976 A general method for numerically simulating the stochastic time evolution of coupled chemical reactions. J. Comp. Phys. 22, 403-434.

[37] Rao, C.V., Wolf, D.M. and Arkin, A.P., 2002. Control, exploitation and tolerance of intracellular noise. Nature, 420, 231-237.

[38] Raj, A. and Van Oudenaarden, A., 2008. Nature, nurture, or chance: stochastic gene expression and its consequences. Cell, 135(2), pp.216-226.

[39] Eldar, A. and Elowitz, M.B., 2010. Functional roles for noise in genetic circuits. Nature, 467, 167-173.

[40] Balázsi, G., van Oudenaarden, A. \& Collins, J.J., 2011. Cellular decision making and biological noise: from microbes to mammals. Cell, 144, 910-925.

[41] Howard J., and Hyman, A.A. 2003. Dynamics and mechanics of the microtubule plus end. Nature 422:753-758.

[42] Antal, T, Krapivsky, P, Redner, S, Mailman, M and Chakraborty, B. 2007a. Dynamics of an idealized model of microtubule growth and catastrophe. Physical Review E 76, 041907.

[43] Antal, T, Krapivsky, P. and Redner, S, 2007b. Dynamics of Microtubule Instabilities. J Stat Mech. 1, L05004.

[44] Solé R. 2011. Phase transitions. Princeton U. Press, Princeton, NJ.

[45] Adler, J., 1976. The sensing of chemicals by bacteria. Sci.
Am. 234, 40-47.

[46] Eisenbach, M., 2001. Bacterial chemotaxis. Encyclopedia Life Sci.

[47] Schweitzer, F. 2003. Brownian agents and active particles. Springer, Berlin.

[48] Hu, D.L., Phonekeo, S., Altshuler, E. and BrochardWyart, F., 2016. Entangled active matter: From cells to ants. Europ. Phys. J, 225, 629-649.

[49] Chien, T., Doshi, A. and Danino, T., 2017. Advances in bacterial cancer therapies using synthetic biology. Curr. Opin. Syst. Biol. 5, 1-8.

[50] Wu, M.R., Jusiak, B. and Lu, T.K., 2019. Engineering advanced cancer therapies with synthetic biology. Nature Rev. Cancer 19, 187-195.

[51] Din, M.O., Danino, T., Prindle, A. et al. 2016. Synchronized cycles of bacterial lysis for in vivo delivery. Nature $536,81-85$.

[52] Kauffman, S.A., 1969. Metabolic stability and epigenesis in randomly constructed genetic nets. J. Theor. Biol. 22, 437-467.

[53] Kauffman, S.A., 1993. Origins of order. Oxford, New York.

[54] Jacob, F. and Monod, J., 1963. Genetic repression, allosteric inhibition, and cellular differentiation. In Cytodifferentiation and macromolecular synthesis (Vol. 21, pp. 30-64). Academic Press. New York.

[55] Serra, R., M. Villani, A. Graudenzi, and S. Kauffman, 2007, Why a simple model of genetic regulatory networks describes the distribution of avalanches in gene expression data. J. Theor. Biol. 246, 449.

[56] Balleza E., Alvarez-Buylla E.R., Chaos A., Kauffman S., Shmulevich I., Aldana M. 2008. Critical dynamics in genetic regulatory networks: examples from four kingdoms. PLoS One 3(6): e2456.

[57] Daniels, B.C., Kim, H., Moore, D. et al. 2018. Criticality distinguishes the ensemble of biological regulatory networks. Physical review letters 121, 138102.

[58] Solé R., Amor D.R., Duran-Nebreda S., Conde-Pueyo N., Carbonell-Ballestero M., Montañez. 2016. Synthetic collective intelligence. BioSys 148, 47-61.

[59] Davidescu, M. and Couzin, I., 2014. Transient Leadership and Collective Cell Movement in Early Diverged Multicellular Animals. arXiv:1407.0972.

[60] Smith, C. and Mayorova, T., 2019. Insights into the evolution of digestive systems from studies of Trichoplax adhaerens. Cell and Tissue Research $\mathbf{3 7 7}$.

[61] Blackiston, D., Lederer, E., Kriegman, S., Garnier, S., Bongard, J. and Levin, M., 2021. A cellular platform for the development of synthetic living machines. Science Robotics, 6(52).

[62] Ebrahimkhani, M.R. and Levin, M., 2021. Synthetic living machines: A new window on life. Iscience, p.102505.

[63] Solé, R., 2016. Synthetic transitions: towards a new synthesis. Philosophical Transactions of the Royal Society B 371, 20150438. 